\title{
The monocyte chemoattractant protein-1 (MCP-1)/CCR2 system is involved in peritoneal dialysis-related epithelial-mesenchymal transition of peritoneal mesothelial cells
}

\author{
Sun Ha Lee ${ }^{1,5}$, Hye-Young Kang ${ }^{1,5}$, Kyung Sik Kim², Bo Young Nam¹, Jisun Paeng ${ }^{1}$, Seonghun Kim¹, Jin Ji Li ${ }^{1,3}$, \\ Jung Tak Park', Dong Ki Kim ${ }^{4}$, Seung Hyeok Han ${ }^{1}$, Tae-Hyun Yoo ${ }^{1}$ and Shin-Wook Kang ${ }^{1}$
}

Epithelial-mesenchymal transition (EMT) of peritoneal mesothelial cells (PMCs) has a role in the process of peritoneal fibrosis (PF), a serious complication in peritoneal dialysis (PD) patients. Even though monocyte chemoattractant protein-1 (MCP-1) was demonstrated to directly increase extracellular matrix (ECM) synthesis, the role of the MCP-1/CCR2 system in PD-related EMT and ECM synthesis in cultured human PMCs (HPMCs) and in an animal model of PD has never been elucidated. In vitro, HPMCs were exposed to $5.6 \mathrm{mM}$ glucose (NG), NG + MCP-1 (10 ng/ml) (NG + MCP-1), or $100 \mathrm{mM}$ glucose (HG) with or without CCR2 inhibitor (RS102895) (CCR2i) or a dominant-negative mutant MCP-1-expressing lentivirus (LV-mMCP-1). In vivo, PD catheters were inserted into 60 Sprague-Dawley rats, and saline (Control, C) $(N=30)$ or 4.25\% PD solution (PD) $(N=30)$ was infused for 4 weeks. Twenty rats from each group were treated with empty LV or LVmMCP-1 intraperitoneally. Snail, E-cadherin, $\alpha$-smooth muscle actin ( $\alpha$-SMA), and fibronectin protein expression in HPMCs and the peritoneum was evaluated by western blot analysis. Compared with NG cells, Snail, $\alpha$-SMA, and fibronectin expression was significantly increased, while E-cadherin expression was significantly decreased in HPMCs exposed to HG and NG + MCP-1, and these changes were significantly abrogated by CCR2i $(P<0.05)$. In addition, MCP-1-induced EMT was significantly attenuated by anti-TGF- $\beta 1$ antibody. In PD rats, Snail and fibronectin expression was significantly increased in the peritoneum, whereas the ratios of E-cadherin/ $\alpha$-SMA protein expression were significantly decreased $(P<0.05)$. The thickness of the peritoneum and the intensity of Masson's trichrome staining in the peritoneum were also significantly higher in PD rats than in $C$ rats $(P<0.05)$. These changes in PD rats were significantly abrogated by LV-mMCP-1. These findings suggest that the MCP-1/CCR2 system is directly involved in PD-related EMT and ECM synthesis and that this is mediated, at least in part, via TGF- $\beta 1$.

Laboratory Investigation (2012) 92, 1698-1711; doi:10.1038/labinvest.2012.132; published online 24 September 2012

KEYWORDS: epithelial-mesenchymal transition; monocyte chemoattractant protein-1; peritoneal fibrosis; peritoneal mesothelial cells; transforming growth factor- $\beta 1$

Continuous ambulatory peritoneal dialysis (CAPD) has been used as a renal replacement therapy in end-stage renal disease (ESRD) patients, along with hemodialysis. ${ }^{1}$ However, after long-term treatment with CAPD, ultrafiltration failure and reduced small solute clearance due to peritoneal fibrosis (PF) lead to inadequacy or even termination of PD in some patients. ${ }^{2}$ Therefore, there is an imperative necessity for the understanding of the molecular pathogenesis of $\mathrm{PF}$ and the identification of targets for effective therapy to prevent PF.

$\mathrm{PF}$ is pathologically characterized by a denudation of mesothelial cells from the peritoneum, an increase in capillary number, and submesothelial deposition of extracellular

\footnotetext{
${ }^{1}$ Department of Internal Medicine, College of Medicine, Brain Korea 21, Severance Biomedical Science Institute, Yonsei University, Seoul, Korea; ${ }^{2}$ Department of Surgery, College of Medicine, Brain Korea 21, Yonsei University, Seoul, Korea; ${ }^{3}$ Nephrology and Dialysis Unit, Department of Internal Medicine, The Affiliated Hospital, YanBian University Medical College, JiLin, China and ${ }^{4}$ Department of Internal Medicine, Seoul National University Hospital, Seoul, Korea

Correspondence: Professor S-W Kang, MD, PhD, Department of Internal Medicine, College of Medicine, Severance Biomedical Science Institute, Yonsei University, 134 Shinchon-Dong, Seodaemoon-Gu, Seoul 120-752, Korea.

E-mail: kswkidney@yuhs.ac
}

${ }^{5}$ These two authors contributed equally to this work. 
matrix $(\mathrm{ECM}) .^{3}$ In the past, resident stromal fibroblasts and inflammatory cells were considered to be the principle cells responsible for PF, but accumulating evidence has suggested that peritoneal mesothelial cells (PMCs) also contribute to the development of peritoneal structural changes in CAPD patients through epithelial-mesenchymal transition (EMT) ${ }^{4}$

EMT is a biologic process that allows a polarized epithelial cell, which normally interacts with basement membrane via its basal surface, to undergo multiple biochemical changes that enable it to assume a mesenchymal cell phenotype, which includes enhanced migratory capacity, invasiveness, elevated resistance to apoptosis, and greatly increased production of ECM components. ${ }^{5}$ The EMT starts with the dissociation of intercellular junctions, as a result of downregulation of adhesion molecules such as E-cadherin through the induction of the transcriptional repressor Snail. Then, the cells adopt a front-back polarity, as a result of cytoskeleton reorganization, and acquire $\alpha$-smooth muscle actin ( $\alpha$-SMA) expression and increased migratory capacity. In the latest stages of EMT, the cells acquire the capacity to degrade the basement membrane, to invade the fibrotic stroma, and to produce ECM components such as fibronectin and collagen. ${ }^{5,6}$ Numerous previous studies have demonstrated that EMT is involved in a variety of normal physiologic processes, including embryo implantation, embryogenesis, and organ development, as well as pathologic processes such as cancer metastasis and fibrotic disorders. ${ }^{5}$ In addition, mounting evidence has indicated that TGF- $\beta 1$ is a master inducer of EMT of various cells including PMCs. Myofibroblastic conversion was observed in cultured human PMCs (HPMCs) exposed to TGF- $\beta 1,{ }^{7}$ and transition of mesothelial cells and appearance of epithelial cells in the submesothelial zone were demonstrated in the peritoneum of rats after intraperitoneal injection of an adenovirus vector that transfers active TGF- $\beta 1 .^{8}$

Monocytes/macrophages are the principle cells found at sites of inflammation. These cells extravasate from the bloodstream through a process mediated by chemokines secreted from resident cells. Chemokines are a family of chemotactic cytokines that induce the migration of various cell types; to date, $>40$ chemokines have been identified. ${ }^{9}$ Among these, monocyte chemoattractant protein (MCP)- 1 is the most extensively studied. MCP-1 has been shown to have an important role in the pathogenesis of various inflammatory and fibrotic diseases, including diabetic nephropathy, ${ }^{10}$ renal fibrosis, ${ }^{11}$ and cardiac fibrosis. ${ }^{12}$ MCP-1 has also been suggested to have a functional role in the initiation and progression of $\mathrm{PF}$ via the recruitment and activation of monocytes/macrophages, which are known to release profibrotic cytokines such as TGF- $\beta 1$ and fibroblast growth factor, which in turn modifies the biology of resident cells. ${ }^{13}$ Recently, however, the direct involvement of the MCP-1/CCR2 system has been demonstrated in numerous pathological conditions. ${ }^{14-16}$ In addition, several recent studies have shown that there may be an intrinsic regulatory loop between MCP-1 and TGF- $\beta 1 .{ }^{17-22}$ To our knowledge, however, no study has investigated if there is a direct association between the MCP-1/ CCR2 system and TGF- $\beta 1$ in PMCs. Moreover, the direct effect of MCP-1 on EMT and ECM synthesis has never been elucidated in these cells. In this study, therefore, we investigated the effect of MCP-1 on EMT and fibronectin expression in cultured HPMCs and whether high glucose increased MCP-1 production, which in turn stimulated TGF$\beta 1$-induced EMT, in these cells. The effects of MCP-1/CCR2 inhibition on EMT and PF were also examined in an animal model of PD.

\section{MATERIALS AND METHODS Isolation of HPMCs}

HPMCs were isolated according to the method described by Stylianou et al. ${ }^{23}$ Briefly, a piece of human omentum, obtained from consenting patients who underwent elective abdominal surgery, was washed three times with sterile phosphate-buffered saline (PBS) and incubated in $0.05 \%$ trypsin- $0.02 \%$ EDTA solution for $20 \mathrm{~min}$ at $37^{\circ} \mathrm{C}$ with continuous shaking. After incubation, the suspension containing free HPMCs was centrifuged at $100 \mathrm{~g}$ for $10 \mathrm{~min}$ at $4{ }^{\circ} \mathrm{C}$. The cell pellet was then washed once and resuspended in M199 medium supplemented with 10\% fetal bovine serum (FBS), $100 \mathrm{U} / \mathrm{ml}$ penicillin, $100 \mathrm{mg} / \mathrm{ml}$ streptomycin, and $26 \mathrm{mM} \mathrm{NaHCO}$, and seeded onto culture dishes. In this study, a total of four different patients' HPMCs were used and all these four different sets of HPMCs showed similar responses.

\section{Inhibition of the MCP-1/CCR2 System}

In this study, two different methods were utilized to inhibit the MCP-1/CCR2 system in HPMCs, a specific chemical inhibitor of CCR2 (RS102895) (Sigma Chemical Co., St Louis, MO, USA) and mutant MCP-1 (mMCP-1), which we used to competitively inhibit native MCP-1 action. The mMCP-1 gene was constructed by recombinant PCR using wild-type human MCP-1 cDNA from HPMCs as a template, and the mMCP-1 amplicon was then cloned into the BamHI $\left(5^{\prime}\right)$ and XhoI $\left(3^{\prime}\right)$ sites of the eukaryotic expression vector plasmid cDNA3 (Invitrogen, Carlsbad, CA, USA) as previously described. ${ }^{24}$

\section{Generation of mMCP-1-Expressing Lentivirus}

Human embryonic kidney (HEK) 293FT cells (Invitrogen) were transfected with the expression vector and two helper plasmids, namely packaging plasmid pCMV $\Delta 8.9$ and vesicular stomatitis virus $\mathrm{G}$ protein plasmid, at $1,7.5$, and $5.5 \mu \mathrm{g}$ of DNA per $150 \mathrm{~mm}$ plate, using the calcium phosphate method. After $72 \mathrm{~h}$, supernatants from four plates were pooled, centrifuged at $780 \mathrm{~g}$ for $5 \mathrm{~min}$, and filtered through a $0.45-\mu \mathrm{m}$ pore size filter. Next, centrifugation was performed at $83000 \mathrm{~g}$ for $1.5 \mathrm{~h}$, and the resulting pellet was resuspended in $100 \mu \mathrm{l}$ of PBS. Lentivirus titers were determined by transfecting HEK 293T cells with a dilution series of the viral 
suspension, and lentivirus samples with a titer of $4 \times 10^{8}$ transfection units $/ \mathrm{ml}$ were stored at $-80{ }^{\circ} \mathrm{C}$.

The aforementioned mMCP-1 construct was cloned into the lentiviral transfer vector FCK-Halo-GFP (Addgene, Cambridge, MA, USA) using NheI/AgeI to create a mMCP-1 lentivirus vector.

\section{HPMC Experiments}

Subconfluent HPMCs were serum restricted for $24 \mathrm{~h}$, and the medium was then changed to serum-free M199 medium containing normal glucose $(5.6 \mathrm{mM}, \mathrm{NG}), \mathrm{NG}+$ mannitol (94.4 mM, NG $+\mathrm{M})$, or high glucose $(100 \mathrm{mM}, \mathrm{HG})$ with or without CCR2 inhibitor (1-10 $\mu \mathrm{M}$ RS102895) and TGF- $\beta 1$ neutralizing antibody $(25 \mu \mathrm{g} / \mathrm{ml})$. HPMCs were also exposed to medium containing recombinant human MCP-1 $(10 \mathrm{ng} / \mathrm{ml})$ or TGF- $\beta 1(2 \mathrm{ng} / \mathrm{ml})$ (R\&D Systems, Minneapolis, MN, USA) with or without TGF- $\beta 1$ neutralizing antibody and CCR2 inhibitor, respectively. For lentiviral transfection, $0.7 \mathrm{ml}$ of the lentivirus suspension containing a variable amount of mMCP-1 (final concentrations: 0.5 and $1.0 \mu \mathrm{g} / \mathrm{ml}$ ) was added to cultured HPMCs. After $48 \mathrm{~h}$, the medium was changed to routine culture medium and HPMCs were cultured for an additional 3 days. After serum restriction for $24 \mathrm{~h}$, transfected HPMCs were exposed to NG or NG + MCP-1 media. At $72 \mathrm{~h}$ after the media change, cells were harvested and conditioned media were collected. The doses of MCP- 1 and TGF- $\beta 1$ used in the experiments were determined based on the results of preliminary experiments.

\section{Animal Studies}

All animal studies were conducted under an approved protocol. Peritoneal access ports were inserted in 60 male Sprague-Dawley rats weighing $250-280 \mathrm{~g}$, and $2 \mathrm{ml}$ of saline with $1 \mathrm{IU} / \mathrm{ml}$ heparin was instilled intraperitoneally until wound healing. One week after surgery, 30 rats received a daily (once per day) $20 \mathrm{ml}$ of saline instillation and 30 rats were instilled daily with $20 \mathrm{ml}$ of standard PD fluid (Dianeal $\mathrm{PD}-2$ peritoneal dialysis solution with $4.25 \%$ dextrose, $\mathrm{pH}$ 5.2, Baxter Healthcare Ltd., Singapore) for 4 weeks. Twenty rats from each group were treated with empty lentivirus vector (LV-empty) or lentivirus vector containing mMCP-1 (LV-mMCP-1) intraperitoneally via the peritoneal access port at a dose of $4 \times 10^{8}$ transfection units every 5 days three times, while ten rats in each group were left untreated (C, PD). After 4 weeks of PD, dialysate in the abdominal cavity was collected, and then the abdomen was opened by a midline incision and the entire anterior abdominal wall was removed at the contralateral side to the tip of the implanted catheter. One fifth of the whole tissue adjacent to the liver was fixed in $10 \%$ neutral-buffered formalin for pathologic examination, while the parietal peritoneum dissected from the major part of the tissue was washed in ice-cold PBS, snap-frozen in liquid nitrogen, pulverized with a mortar and pestle while frozen, and suspended in SDS sample buffer (2\% SDS, 10 mM Tris-HCl, pH 6.8, 10\% (vol/vol) glycerol). After a centrifugation at $16000 \mathrm{~g}$ for $15 \mathrm{~min}$ at $4{ }^{\circ} \mathrm{C}$, the supernatant was kept at $-80{ }^{\circ} \mathrm{C}$ until use.

\section{ELISA}

The levels of MCP- 1 and TGF- $\beta 1$ in conditioned media and dialysate were determined using commercial ELISA kits (R\&D Systems) according to manufacturer's protocols as previously described. ${ }^{17}$ The kits for human MCP-1 were species specific and sensitive up to $5.0 \mathrm{pg} / \mathrm{ml}$. The minimum sensitivity for human TGF- $\beta 1$ of the kit used in this study was $4.6 \mathrm{pg} / \mathrm{ml}$.

\section{Western Blot Analysis}

The protein expression of MCP-1, CCR2, Snail, E-cadherin, $\alpha$-SMA, and fibronectin in HPMCs and peritoneal tissue was evaluated by western blot as previously described. ${ }^{18}$ The band densities were measured using TINA image software (Raytest, Straubenhardt, Germany), and the changes in the optical densities of bands from the treated groups relative to NG cells or $\mathrm{C}$ peritoneum were used in the analysis.

\section{Immunohistochemistry and Masson's Trichrome Staining}

The peritoneum samples were fixed in 10\% neutral-buffered formalin, processed in the standard manner, and $5 \mu \mathrm{m}$-thick sections of paraffin-embedded tissues were utilized for immunohistochemical staining. Slides were deparaffinized, hydrated in ethyl alcohol, and washed in tap water. Antigen retrieval was carried out in $10 \mathrm{mM}$ sodium citrate buffer for 20 min using a Black \& Decker vegetable steamer. Primary antibodies for E-cadherin, $\alpha$-SMA, and fibronectin were diluted to the appropriate concentrations with $2 \%$ casein in bovine serum albumin and then added to the slides, followed by an overnight incubation at $4{ }^{\circ} \mathrm{C}$. After washing, a secondary antibody was added for $20 \mathrm{~min}$, and the slides were washed and incubated with a tertiary PAP complex for $20 \mathrm{~min}$. Diaminobenzidine was added for $2 \mathrm{~min}$ and the slides were counterstained with hematoxylin. Masson's trichrome staining was also performed. A semiquantitative score of staining intensity was determined by examining at least five fields of the peritoneum in each section under $\times 400$ magnification and by digital image analysis (MetaMorph version 4.6r5; Universal Imaging Corp., Downingtown, PA, USA). The score was obtained by multiplying the intensity staining $(0-4+)$ by the percentage of observed area staining for that intensity and these numbers were then added for each experimental animal to give the staining score; $\Sigma$ (intensity of staining $) \times(\%$ of observed area with that intensity) $=$ staining score. The scores form $\mathrm{C}$ rats were assigned a relative value of 100 . The thickness of peritoneum, which was defined as the tissue between the mesothelial surface and the underlying muscle or parenchyma, was assessed as previously described. ${ }^{25}$ Briefly, the maximal thickness of the peritoneum was measured in three Masson's trichrome-stained tissue sections per rats and five 
fields, the center of which included the area of maximal thickness, were examined under $\times 400$ magnification. Areas and perimeter lengths of the peritoneum were obtained from drawn outlines and the average thickness was calculated from rectangular approximation based on the values for area and perimeter in each field of view.

\section{Statistical Analysis}

All values are expressed as means \pm standard errors of the mean (s.e.m.). Statistical analyses were performed using the statistical package SPSS for Windows Ver. 11.0 (SPSS, Inc., Chicago, IL, USA). Results were analyzed using one-way ANOVA with a post hoc Bonferonni's test for multiple comparisons. $P$-values $<0.05$ were considered statistically significant.

\section{RESULTS}

\section{The MCP-1/CCR2 System is Involved in HG-Induced EMT} and Fibronectin Expression in HPMCs

In this study, to first elucidate the presence of CCR2 in HPMCs, western blot for CCR2 protein was performed. As seen in Figure 1a, CCR2 protein expression was demonstrated in cultured HPMCs and its expression was not significantly changed by HG. Second, we examined whether the MCP-1/CCR2 system was involved in EMT and fibronectin expression in HPMCs. To determine the dose of MCP-1 for all subsequent in vitro experiments, HPMCs were cultured under NG media containing various concentrations of MCP-1. Snail protein expression started to be increased by $5.0 \mathrm{ng} / \mathrm{ml} \mathrm{MCP-1}$ and was significantly increased in HPMCs exposed to MCP-1 at a dose higher than $10.0 \mathrm{ng} / \mathrm{ml}$. However, there was no significant difference in Snail protein expression between HPMCs exposed to 10 and $20 \mathrm{ng} / \mathrm{ml}$ MCP-1 $(P<0.05) \quad$ (Figure 1b). The administration of $10 \mathrm{ng} / \mathrm{ml} \mathrm{MCP-1}$ also significantly increased the protein expression of $\alpha$-SMA and fibronectin in HPMCs and significantly decreased E-cadherin protein expression $(P<0.01)$, and these changes were significantly attenuated by blocking CCR2 with $10 \mu \mathrm{M}$ RS102895 $(P<0.01)$, suggesting that MCP-1 directly induces EMT and fibronectin expression in HPMCs via CCR2 (Figure 2a). Next, the role of the MCP-1/ CCR2 system in HG-induced EMT and fibronectin expression was evaluated in HPMCs. Snail and fibronectin protein expression was significantly higher $(P<0.01$ or $P<0.05)$ and the ratios of $\mathrm{E}$-cadherin/ $\alpha$-SMA were significantly lower $(P<0.005)$ in HPMCs cultured under HG conditions than in NG cells, and these changes were significantly abrogated by CCR2 inhibitor treatment $(P<0.01$ or $P<0.05)$ (Figure 2a). In addition, the proportion of fibroblast-like phenotypic cells was significantly higher in MCP-1- and HG-treated HPMCs, and these changes were significantly inhibited by CCR2 inhibitor (Figure $2 \mathrm{~b}$ ). These findings suggested that HG-induced EMT and fibronectin expression was mediated by the MCP-1/CCR2 system. On the other hand, mannitol used as an osmotic control had no effect on EMT and fibronectin
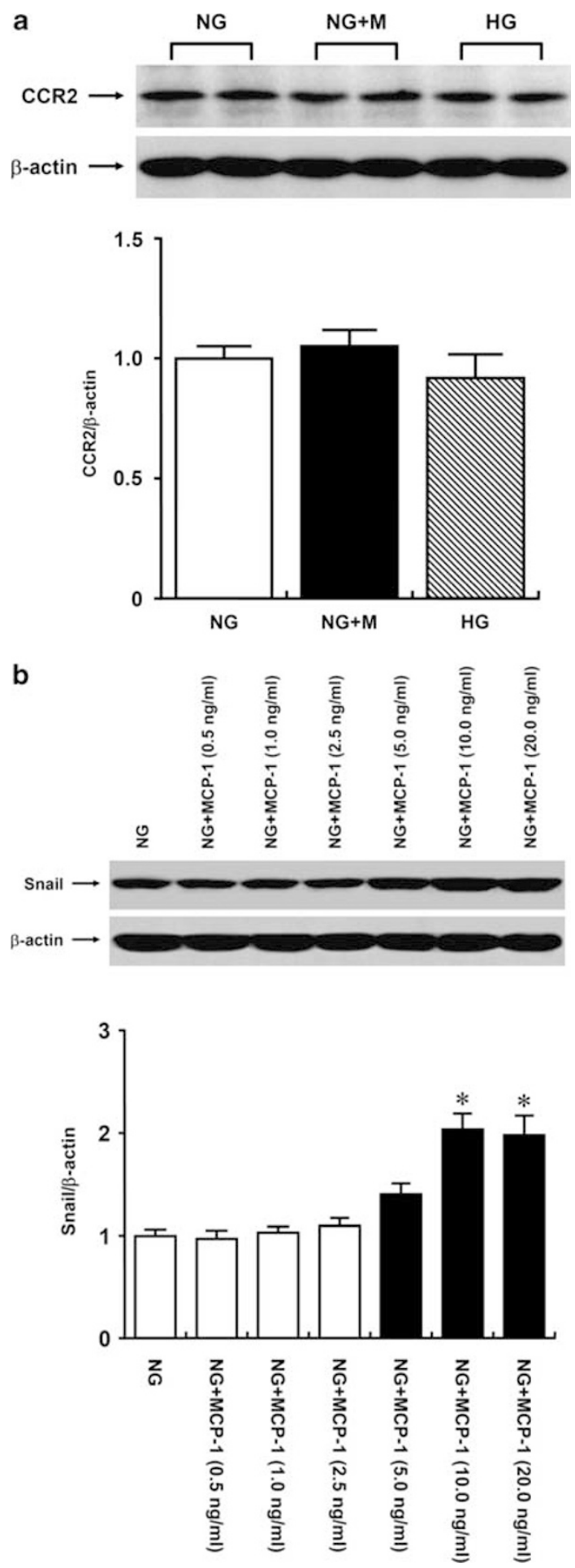

Figure 1 (a) CCR2 protein expression in HPMCs exposed to $5.6 \mathrm{mM}$ glucose (NG), NG +94.4 mM mannitol (NG + M), or $100 \mathrm{mM}$ glucose (HG) (a representative of four western blots). CCR2 protein expression was demonstrated in cultured HPMCs and its expression was not significantly changed by HG. (b) Snail protein expression exposed to various concentrations of MCP-1 $(0.5-20.0 \mathrm{ng} / \mathrm{ml})$ (a representative of four western blots). Snail protein expression started to be increased by $5.0 \mathrm{ng} / \mathrm{ml} \mathrm{MCP-1}$ and was significantly increased in HPMCs exposed to MCP-1 at a dose higher than $10.0 \mathrm{ng} / \mathrm{ml}$. However, there was no significant difference in Snail protein expression between HPMCs exposed to 10 and $20 \mathrm{ng} / \mathrm{ml}$ MCP-1. ${ }^{*} P<0.05$ vs NG. 
expression in HPMCs (Figures 1 and 2a, b). Finally, to determine whether mMCP-1-expressing lentivirus could inhibit the effect of MCP-1 in HPMCs, EMT and fibronectin expression was elucidated in MCP-1-treated HPMCs transfected with LV-mMCP-1 or LV-empty. The significant increase in the expression of Snail, $\alpha$-SMA, and fibronectin and the significant decrease in E-cadherin protein expression in HPMCs treated with MCP-1 were significantly ameliorated by LV-mMCP-1 $(P<0.01$ or $P<0.05)$. However, LV-empty had no effect on Snail, E-cadherin, $\alpha$-SMA, and fibronectin protein expression in HPMCs (Figure 2c). These findings implied that mMCP-1-expressing lentivirus effectively inhibited the action of MCP-1 on EMT and fibronectin expression in HPMCs.

\section{The TGF- $\beta 1$ System is Involved in HG-Induced MCP-1 levels, EMT, and Fibronectin Expression in HPMCs}

To clarify whether HG increased MCP-1 expression, MCP-1 levels were determined in culture conditioned media by ELISA. As seen in Figure 3a, the concentrations of MCP- 1 in TGF- $\beta 1$ - and HG-conditioned media were significantly higher than those in NG media $(P<0.01)$, and these increases in MCP-1 levels were significantly ameliorated by the addition of anti-TGF- $\beta 1$ antibody $(P<0.01$ or $P<0.05)$. In addition, the increases in Snail and fibronectin protein expression and the decrease in the ratios of E-cadherin/ $\alpha$-SMA in HG-treated HPMCs were significantly ameliorated by $25 \mu \mathrm{g} / \mathrm{ml}$ TGF- $\beta 1$ neutralizing antibody $(P<0.01$ or $P<0.05)$ (Figure $3 \mathrm{~b}$ ). These findings implied that HG induced MCP-1, EMT, and fibronectin expression in HPMCs via the TGF- $\beta 1$ system. On the other hand, there was no significant change in MCP-1 levels in mannitol-conditioned media (Figure 3a).

\section{The MCP-1/CCR2 System is Involved in HG-Induced TGF- $\beta 1$ Levels}

Since TGF- $\beta 1$ is the best-characterized cytokine implicated in the process of EMT, we attempted to elucidate whether the MCP-1/CCR2 system was involved in HG-induced TGF- $\beta 1$ levels. First, the direct effect of MCP- 1 on TGF- $\beta 1$ concentrations was explored. The administration of $10 \mathrm{ng} / \mathrm{ml}$
MCP-1 significantly increased the levels of TGF- $\beta 1$ in conditioned media $(P<0.05)$, and this increase in TGF- $\beta 1$ concentrations was significantly attenuated by the addition of CCR2 inhibitor $(P<0.05)$ (Figure 4$)$. Second, the role of the MCP-1/CCR2 system in HG-induced TGF- $\beta 1$ levels was examined. Compared with NG media, the concentrations of TGF- $\beta 1$ were significantly higher in HG-conditioned media $(2.06 \pm 0.08$ vs $3.34 \pm 0.21 \mathrm{ng} / \mathrm{ml}, P<0.05)$, and this HG-induced increase in TGF- $\beta 1$ levels was significantly abrogated by the administration of CCR2 inhibitor $(P<0.05)$ (Figure 4). These findings indicated that HG increased TGF$\beta 1$ concentrations in cultured HPMCs via the MCP-1/CCR2 system. On the other hand, there was no significant change in TGF- $\beta 1$ levels in mannitol-conditioned media (Figure 4).

\section{TGF- $\beta 1$ and MCP-1 Induce EMT and Fibronectin Expression in HPMCs Partly Via the MCP-1/CCR2 System and the TGF- $\beta 1$ Pathway, Respectively}

Next, we clarified whether the MCP-1/CCR2 system and the TGF- $\beta 1$ pathway are involved in TGF- $\beta 1$ - and MCP-1induced EMT and fibronectin expression, respectively. The administration of $2 \mathrm{ng} / \mathrm{ml}$ TGF- $\beta 1$ significantly increased the protein expression of Snail, $\alpha$-SMA, and fibronectin in HPMCs and significantly decreased E-cadherin protein expression, and these changes were significantly attenuated by the inhibition of the MCP-1/CCR2 system with RS102895 $(P<0.01$ or $P<0.05)$ (Figure 5). MCP-1-induced EMT and fibronectin expression were significantly abrogated by the administration of TGF- $\beta 1$ neutralizing antibody $(P<0.01$ or $P<0.05$ ) (Figure 5). These findings suggested that the TGF$\beta 1$-induced EMT and fibronectin expression partly via MCP1/CCR2 system, while MCP-1-induced EMT and fibronectin expression were partly mediated by the TGF- $\beta 1$ pathway.

\section{MCP-1 Expression is Increased in the Peritoneum in a PD Rat Model}

To explore whether MCP-1 was induced in the peritoneum in an animal model of PD, peritoneal MCP-1 expression was assessed by western blot and immunohistochemical staining. As seen in Figure 6a, peritoneal MCP-1 expression was sig-

\footnotetext{
Figure 2 (a) Snail, E-cadherin, $\alpha$-SMA, and fibronectin protein expression in HPMCs exposed to $5.6 \mathrm{mM}$ glucose (NG), NG $+94.4 \mathrm{mM}$ mannitol (NG + M), $\mathrm{NG}+\mathrm{CCR} 2$ inhibitor $(10 \mu \mathrm{M}$ RS102895) (NG + CCR2i), NG + 10 ng/ml MCP-1 (NG + MCP-1), NG + MCP-1 + CCR2i, 100 mM glucose (HG), or HG + CCR2i (a representative of five western blots). MCP-1 increased Snail, $\alpha$-SMA, and fibronectin and significantly decreased E-cadherin protein expression in HPMCs, and these changes were significantly abrogated by CCR2 inhibitor treatment. HG also significantly increased Snail, $\alpha$-SMA, and fibronectin and significantly decreased E-cadherin protein expression in HPMCs, which were significantly ameliorated by CCR2 inhibitor. ${ }^{*} P<0.05$ vs NG, ${ }^{\#} P<0.05$ vs $\mathrm{NG}+\mathrm{MCP}-1$ or HG, ${ }^{\$} P<0.005$ vs NG, ${ }^{\dagger} P<0.01$ vs NG + MCP-1 or HG, ${ }^{+} P<0.01$ vs NG. (b) Morphologic changes of HPMCs exposed to $5.6 \mathrm{mM}$ glucose (NG), NG + 94.4 mM mannitol (NG + M), NG + CCR2 inhibitor (10 $\mu \mathrm{M}$ RS102895) (NG + CCR2i), NG + 10 ng/ml MCP-1 (NG + MCP-1), NG + MCP-1 + CCR2i, $100 \mathrm{mM}$ glucose $(\mathrm{HG})$, or HG + CCR2i. HPMCs showed fibroblast-like phenotypic changes 3 days after MCP-1 and HG treatment, and these changes were reversed by CCR2 inhibitor ( $\times 40$ and $\times 100)$. ${ }^{*} P<0.001$ vs NG, ${ }^{*} P<0.001$ vs NG + MCP-1, ${ }^{\$} P<0.01$ vs HG. (c) Snail, E-cadherin, $\alpha$-SMA, and fibronectin protein expression in HPMCs exposed to $5.6 \mathrm{mM}$ glucose (NG), NG $+10 \mathrm{ng} / \mathrm{ml} \mathrm{MCP-1} \mathrm{(NG} \mathrm{+} \mathrm{MCP-1),} \mathrm{NG} \mathrm{+} \mathrm{MCP-1} \mathrm{+} \mathrm{empty} \mathrm{lentivirus}$ (NG + MCP-1 + LV-empty), or NG + MCP-1 + lentivirus vector containing mMCP-1 (NG + MCP-1 + LV-mMCP-1) (a representative of five western blots). The significant increases in Snail, $\alpha$-SMA, and fibronectin protein expression and the significant decrease in E-cadherin protein expression in MCP-1stimulated HPMCs were significantly ameliorated by mMCP-1-expressing lentivirus but not by empty lentivirus. ${ }^{*} P<0.05$ vs $\mathrm{NG}$, ${ }^{\#} P<0.05$ vs $\mathrm{NG}+\mathrm{MCP}-1$, ${ }^{\$} P<0.005$ vs NG, ${ }^{\dagger} P<0.01$ vs NG + MCP-1, ${ }^{+} P<0.01$ vs NG.
} 

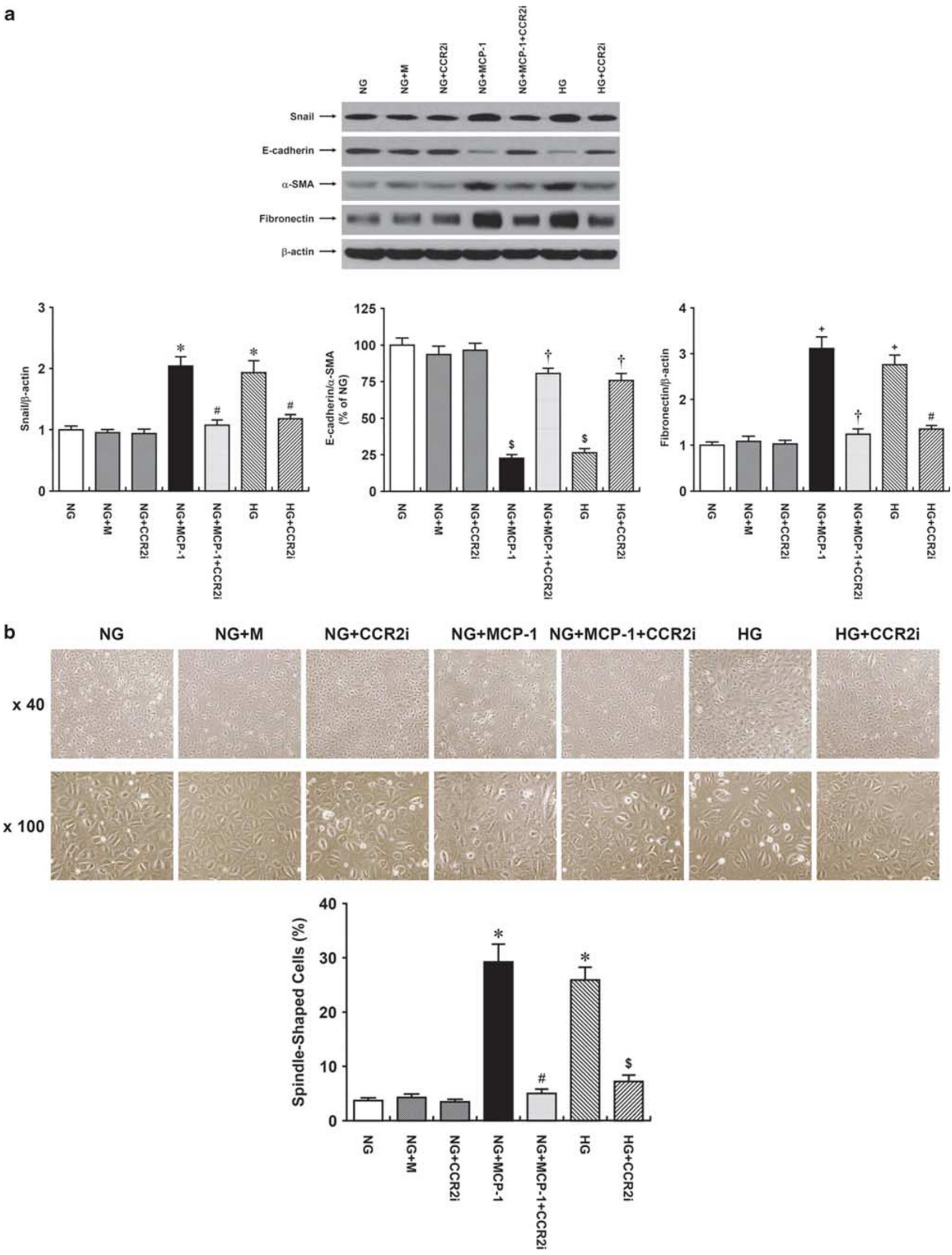

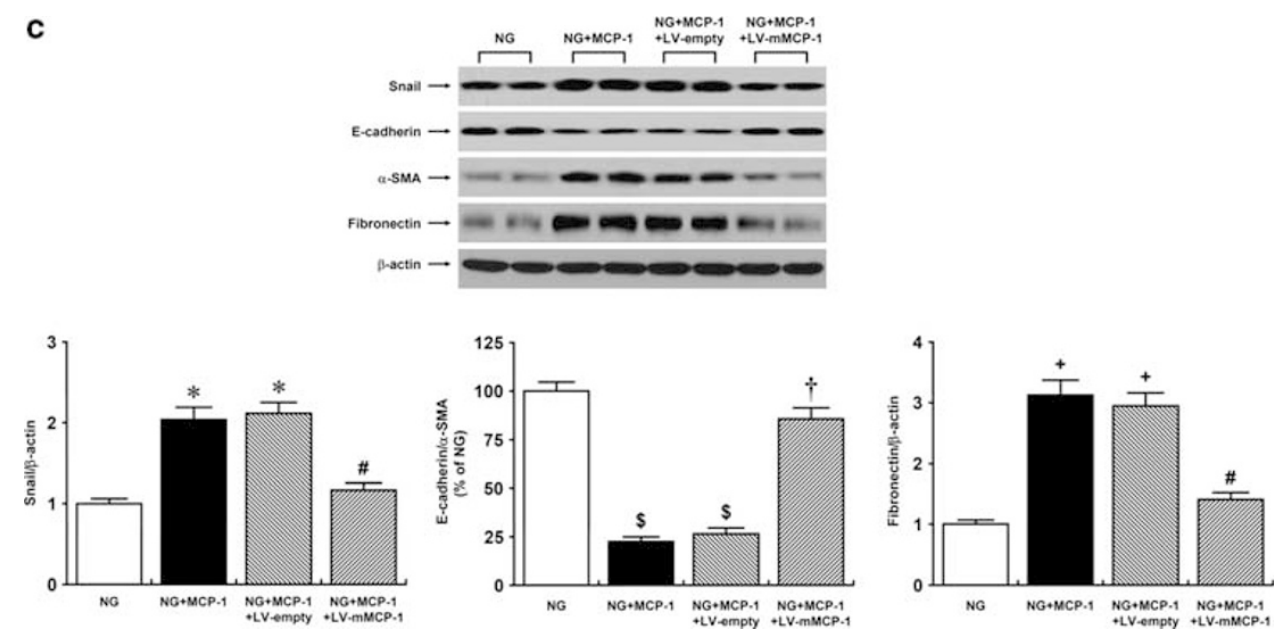

Figure 2 (Continued)

nificantly increased in rats treated with $\mathrm{PD}$ solution compared with $C$ rats $(P<0.05)$, while there was no significant difference in CCR2 protein expression among the groups. The levels of MCP-1 were also significantly higher in the dialysate from rats treated with PD solution $(1.25 \pm 0.14$ vs $8.54 \pm 1.01 \mathrm{ng} / \mathrm{ml}, P<0.001)$. Immunohistochemical staining revealed that the cells responsible for the increase in MCP-1 were fibroblast-like cells and infiltrated inflammatory cells (Figure 6b).

\section{mMCP-1 Lentivirus Inhibits the Peritoneal EMT and ECM Accumulation in a PD Rat Model}

Finally, the impact of the MCP-1/CCR2 system on peritoneal EMT and ECM accumulation was explored in a PD rat model. Snail, $\alpha$-SMA, and fibronectin protein expression was significantly higher, while E-cadherin protein expression was significantly lower in rats treated with PD solution compared with $\mathrm{C}$ rats, and these changes were significantly attenuated by MCP-1 inhibition using a lentivirus system $(P<0.01$ or $P<0.05$ ) (Figure $7 a)$. In contrast, LV-empty had no effect on EMT and fibronectin protein expression in PD rats. Masson's trichrome staining and immunohistochemical staining of the peritoneum also revealed that EMT and fibronectin protein expression were significantly higher and submesothelial layer was significantly thicker in rats treated with $\mathrm{PD}$ solution relative to $\mathrm{C}$ rats, and LV-mMCP-1 significantly abrogated EMT and ECM accumulation in PD rats (Figure 7b). These findings indicated that PD-related EMT and PF were mediated by the MCP-1/CCR2 system.

\section{DISCUSSION}

EMT of PMCs has been implicated as a basic mechanism in the pathogenesis of $\mathrm{PF}$, which is one of the most serious complications of long-term CAPD, leading to membrane failure. ${ }^{2}$ In addition, TGF- $\beta 1$ has been shown to be a key cytokine involved in the process of EMT. ${ }^{26,27}$ In this study, we showed for the first time that the MCP-1/CCR2 system was directly involved in PD-related EMT of PMCs and ECM accumulation. Moreover, we demonstrated that these effects of the MCP-1/CCR2 system were mediated, at least in part, by TGF- $\beta 1$.

EMT is a complex and generally reversible process that has an important role in embryogenesis, ${ }^{28}$ metastasis of malignant cells, ${ }^{29}$ and renal fibrosis. ${ }^{30} \mathrm{~A}$ number of recent studies have demonstrated that PMCs also undergo EMT during $\mathrm{PD}$, leading to $\mathrm{PF}^{4,26,27}$ In the past, resident peritoneal fibroblasts and infiltrating inflammatory cells were considered to be responsible for $\mathrm{PF}^{6}{ }^{6}$ but Yanez-Mo et $a l^{4}$ were the first to show that PMCs underwent a transition from an epithelial phenotype to a mesenchymal phenotype soon after dialysis was initiated and that this was associated with a decrease in the expression of cytokeratins and E-cadherin, suggesting that these cells might also contribute to structural changes during PD. Consistent with most previous studies, the results of this study also showed that E-cadherin expression was decreased, while $\alpha$-SMA expression was increased in HPMCs exposed to HG and in the peritoneum of rats instilled with PD solution, suggesting that PMCs underwent EMT in our in vitro and in vivo experimental models.

Besides its pivotal role in the recruitment and activation of monocytes/macrophages, several recent studies have shown that MCP-1 per se has a direct effect on cell biology. MCP-1 directly increased fibronectin production in cultured mesangial cells. ${ }^{17,19}$ Recently, we also found that MCP-1 directly induced podocyte apoptosis. ${ }^{18}$ In addition, MCP-1 itself induced intercellular adhesion molecule-1 and fibronectin expression in cultured mesangial cells. ${ }^{14}$ Moreover, some investigators have provided evidence that MCP-1 may be directly involved in ECM synthesis using animal models of experimental glomerulonephritis ${ }^{15}$ and diabetic nephropathy. ${ }^{16}$ These findings suggested the possible direct involvement of the MCP-1/CCR2 system in the pathogenesis of PF, which has never been elucidated. In the present study, 

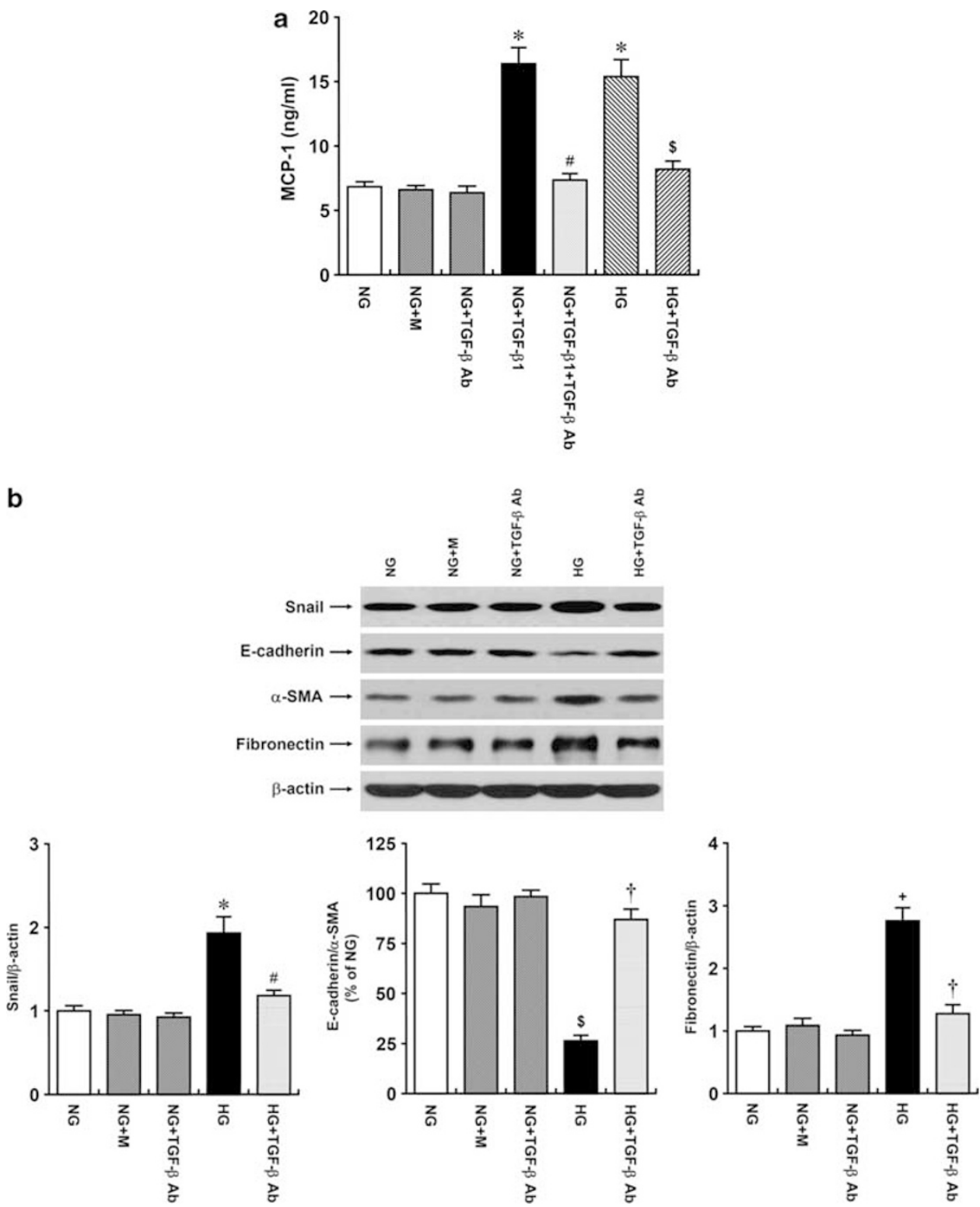

Figure 3 (a) MCP-1 levels in conditioned media of HPMCs exposed to $5.6 \mathrm{mM}$ glucose (NG), NG $+94.4 \mathrm{mM}$ mannitol (NG $+\mathrm{M}$ ), NG $+25 \mu \mathrm{g} / \mathrm{ml}$ TGF- $\beta 1$ neutralizing antibody (NG + TGF- $\beta$ Ab), NG +2 ng/ml TGF- $\beta 1+$ TGF- $\beta$ Ab (NG + TGF- $\beta 1+$ TGF- $\beta$ Ab), 100 mM glucose (HG), or HG + TGF- $\beta$ Ab. There was a 2.4-fold and 2.3-fold increase in MCP-1 concentrations in TGF- $\beta 1$ - and HG-conditioned media compared with NG media, respectively, and treatment with anti-TGF- $\beta 1$ antibody significantly ameliorated HG-induced MCP-1 levels. ${ }^{*} P<0.01$ vs NG, ${ }^{\#} P<0.01$ vs TGF- $\beta 1,{ }^{5} P<0.05$ vs HG. (b) Snail, E-cadherin, $\alpha$-SMA, and fibronectin protein expression in HPMCs exposed to $5.6 \mathrm{mM}$ glucose (NG), NG $+94.4 \mathrm{mM}$ mannitol (NG $+\mathrm{M}$ ), NG $+25 \mu \mathrm{g} / \mathrm{ml}$ TGF- $\beta 1$ neutralizing antibody (NG + TGF- $\beta$ Ab), $100 \mathrm{mM}$ glucose (HG), or HG + TGF- $\beta$ Ab (a representative of five western blots). The increases in Snail and fibronectin protein expression and the decrease in the ratios of E-cadherin/ $\alpha$-SMA in HG-treated HPMCs were significantly attenuated by $25 \mu \mathrm{g} / \mathrm{ml}$ TGF- $\beta 1$ neutralizing antibody. ${ }^{*} P<0.05$ vs NG, ${ }^{\#} P<0.05$ vs $\mathrm{HG},{ }^{\$} P<0.005$ vs NG, ${ }^{\dagger} P<0.01$ vs $\mathrm{HG},{ }^{+} P<0.01$ vs NG.

we found that MCP-1 per se increased fibronectin expression in cultured HPMCs and that HG-induced fibronectin expression was abrogated by the inhibition of the MCP-1/ CCR2 system. The results of this study also demonstrated that $\mathrm{PF}$ was ameliorated in rats on PD by lentivirus-mediated mMCP-1 gene transfer into the peritoneum. On the other hand, even though mMCP-1 was known to competitively inhibit MCP-1 action but not the expression of MCP- $1,{ }^{24}$ the results of this study showed that MCP-1 protein expression was somewhat decreased in PD rats treated with mMCP-1expressing lentivirus. Furthermore, CCR2 protein expression was observed to be slightly increased in PD rats in spite of no significant changes in HG-stimulated HPMCs. Even though it is not clear whether these in vivo consequences are attributed to reduced infiltration of monocytes/macrophages, which express MCP-1 as well as CCR2 and is known to release profibrotic cytokines such as TGF- $\beta 1$ and to control ECM turnover by regulating the balance of various matrix 


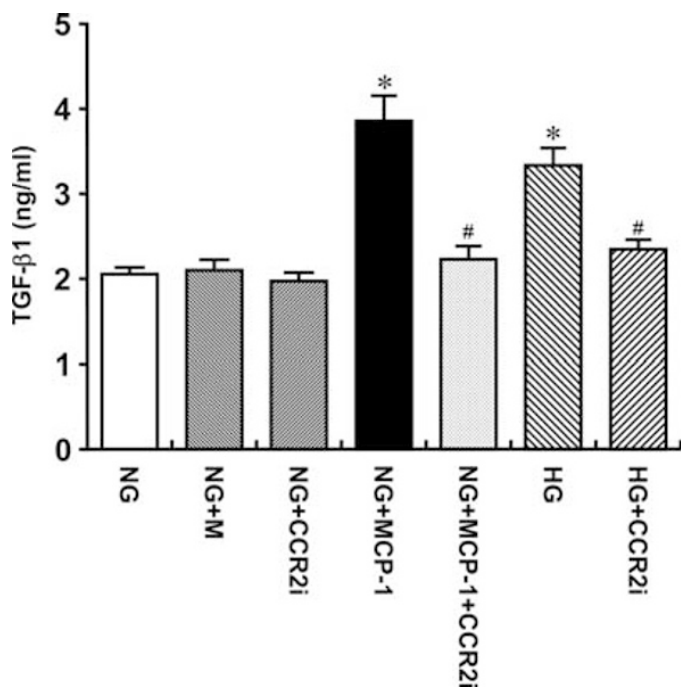

Figure 4 TGF- $\beta 1$ levels in conditioned media of HPMCs exposed to $5.6 \mathrm{mM}$ glucose (NG), NG + $94.4 \mathrm{mM}$ mannitol (NG + M), NG + CCR2 inhibitor (10 $\mu \mathrm{M}$ RS102895) (NG + CCR2i), NG + 10 ng/ml MCP-1 (NG + MCP-1), NG + MCP-1 + CCR2i, $100 \mathrm{mM}$ glucose (HG), or HG + CCR2i. MCP-1 and HG significantly increased TGF- $\beta 1$ concentrations in conditioned media, and these MCP-1- and HG-induced increases in TGF- $\beta 1$ levels were significantly abrogated by CCR2 inhibitor treatment. ${ }^{*} P<0.05$ vs NG, ${ }^{*} P<0.05$ vs NG + MCP-1 or HG. metalloproteinases and tissue inhibitors of matrix metalloproteinases, ${ }^{31}$ or to the impairment of the direct effect of MCP-1 on PMCs, considering the results of our in vitro study, we surmise that the MCP-1/CCR2 system has a direct and pivotal role in ECM accumulation in PD-related PF. Further studies using macrophage-depleted animal or methods to inhibit/control chemotactic property of MCP-1 will be needed to clarify the direct in vivo effect of MCP-1 on the peritoneum. On the other hand, MCP-1 levels in the dialysate of PD rats were significantly higher than those of the previous studies, ${ }^{32-34}$ which ranged from 0.15 to $1.7 \mathrm{ng} / \mathrm{ml}$. We inferred that the divergence of the concentrations of MCP-1 might be attributed to different experimental animals (Wistar rats vs Sprague Dawley rats) and conditions such as the sampling time of the dialysate, study duration, and the amount of infused dialysate. In contrast to most previous studies, which used 2- to 30-min washed sample of abdominal cavity or 90-min indwelled dialysate, at least 6-h indwelled dialysate was used in the current study. Therefore, MCP-1 levels could be much higher due to more concentrated dialysate and more secreted MCP-1 from the peritoneum.

In terms of EMT, no previous study has provided direct evidence that MCP-1 per se induces EMT in any cell type. However, Soria et $a^{35}$ suggested an association between
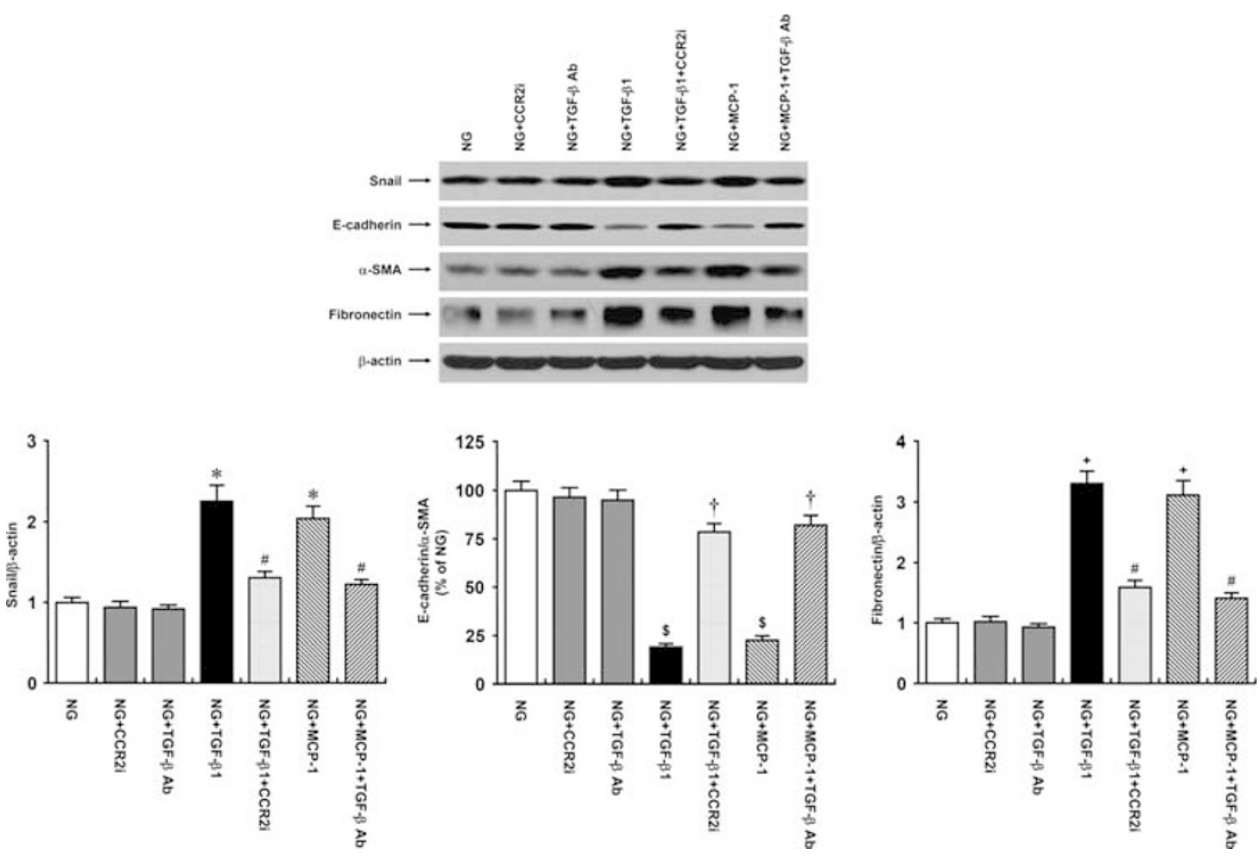

Figure 5 Snail, E-cadherin, $\alpha$-SMA, and fibronectin protein expression in HPMCs exposed to $5.6 \mathrm{mM}$ glucose (NG), NG + CCR2 inhibitor $(10 \mu \mathrm{M}$ RS102895) (NG + CCR2i), NG $+25 \mu \mathrm{g} / \mathrm{ml}$ TGF- $\beta 1$ neutralizing antibody (NG + TGF- $\beta$ Ab), NG $+2 \mathrm{ng} / \mathrm{ml}$ TGF- $\beta 1$ (NG + TGF- $\beta 1$ ),

NG + TGF- $\beta 1+$ CCR2i, NG + $10 \mathrm{ng} / \mathrm{ml} \mathrm{MCP-1}$ (NG + MCP-1), or NG + MCP-1 + TGF- $\beta$ Ab (a representative of five western blots). TGF- $\beta 1$ significantly increased Snail, $\alpha$-SMA, and fibronectin and significantly decreased E-cadherin protein expression in HPMCs compared with NG cells, and these changes were significantly attenuated with CCR2 inhibitor treatment. The protein expression of Snail and fibronectin was also significantly higher, while the ratios of E-cadherin/ $\alpha$-SMA were significantly lower in MCP-1-stimulated HPMCs than in NG cells, and these effects were significantly abrogated by anti-TGF- $\beta 1$ antibody. ${ }^{*} P<0.05$ vs NG, ${ }^{\#} P<0.05$ vs NG + TGF- $\beta 1$ or NG + MCP- $1,{ }^{\$} P<0.005$ vs NG, ${ }^{\dagger} P<0.01$ vs NG + TGF- $\beta 1$ or NG + MCP- 1 , $+P<0.01$ vs NG. 
a

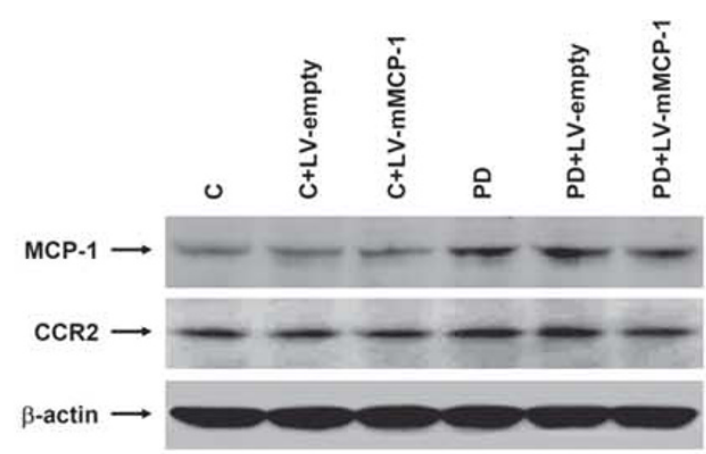

b
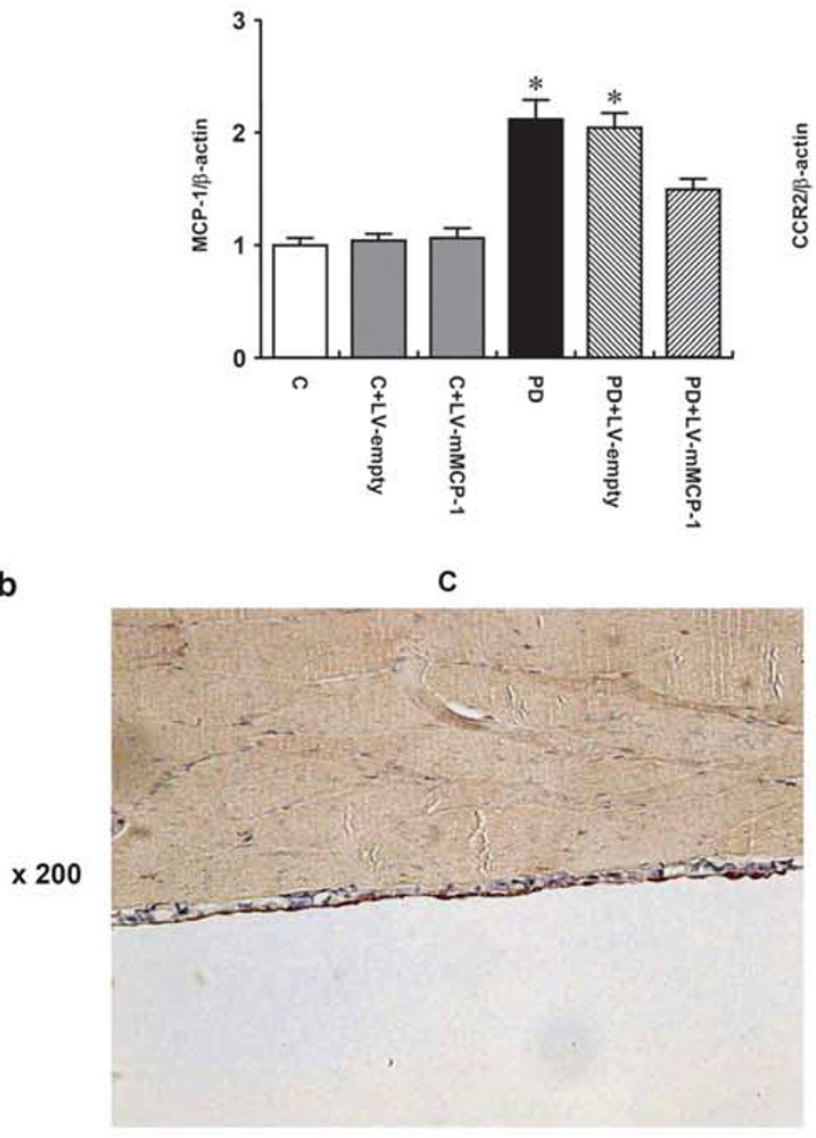

C

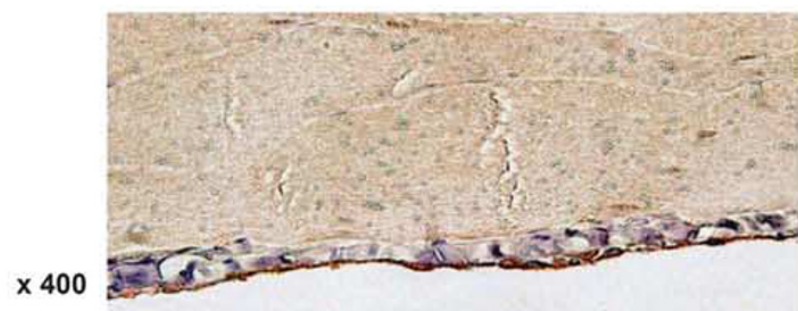

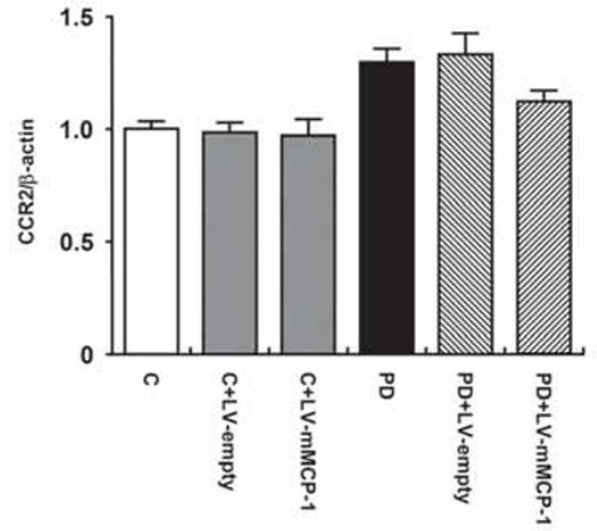

PD
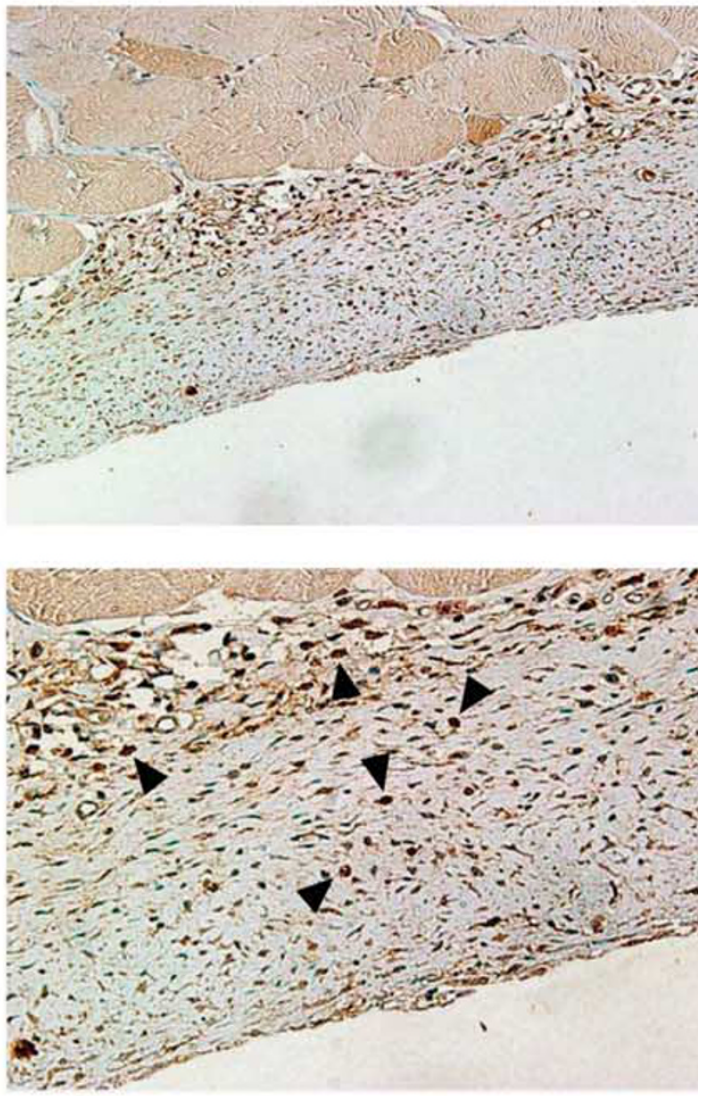

Figure 6 For caption refer page 1708. 
inflammatory mediators and EMT in breast cancer cells. They found that the expression of MCP-1 was related to TNF- $\alpha$ and IL- $1 \beta$ expression and that the continuous stimulation of breast tumor cells by TNF- $\alpha$ and IL- $1 \beta$ led these cells to undergo EMT. In the present study, we demonstrated for the first time that MCP-1 directly decreased E-cadherin expression and increased the expression of $\alpha$-SMA in cultured HPMCs and that inhibition of the MCP-1/CCR2 system attenuated these changes in HG-stimulated HPMCs. In addition, EMT was inhibited by intraperitoneal administration of mMCP-1 in rats on PD. Taken together, the results of this study suggest that the MCP-1/CCR2 system is involved in the process of ECM accumulation in PD-related PF by inducing PMCs to undergo EMT.

Previous studies have shown that there is an intrinsic regulatory loop between the MCP-1/CCR2 system and the TGF- $\beta 1$ pathway. Schneider et al ${ }^{20}$ found that an increase in TGF- $\beta 1$ expression in glomeruli isolated from rats with experimental glomerulonephritis was abrogated by the neutralization of MCP-1. The increase in glomerular TGF$\beta 1$ expression in a model of glomerular immune injury was also inhibited by the administration of anti-MCP-1 antiserum even in the isolated perfused kidney, which was performed to minimize infiltrating inflammatory cells. $^{21}$ In addition, a close interaction between TGF- $\beta 1$ and MCP-1 has also been identified in vitro in cultured mesangial cells and podocytes. ${ }^{17,22}$ Based on these findings, we hypothesized that the impact of the MCP-1/ CCR2 system on EMT of PMCs and ECM accumulation synthesis was associated with the TGF- $\beta 1$ pathway. In the present study, MCP- 1 directly increased TGF- $\beta 1$ production in cultured HPMCs and MCP-1-induced EMT and fibronectin expression were ameliorated by anti-TGF- $\beta 1$ antibody. Moreover, HG-induced EMT and ECM accumulation were attenuated by MCP-1/CCR2 inhibition both in vitro and in vivo, along with reduced TGF- $\beta 1$
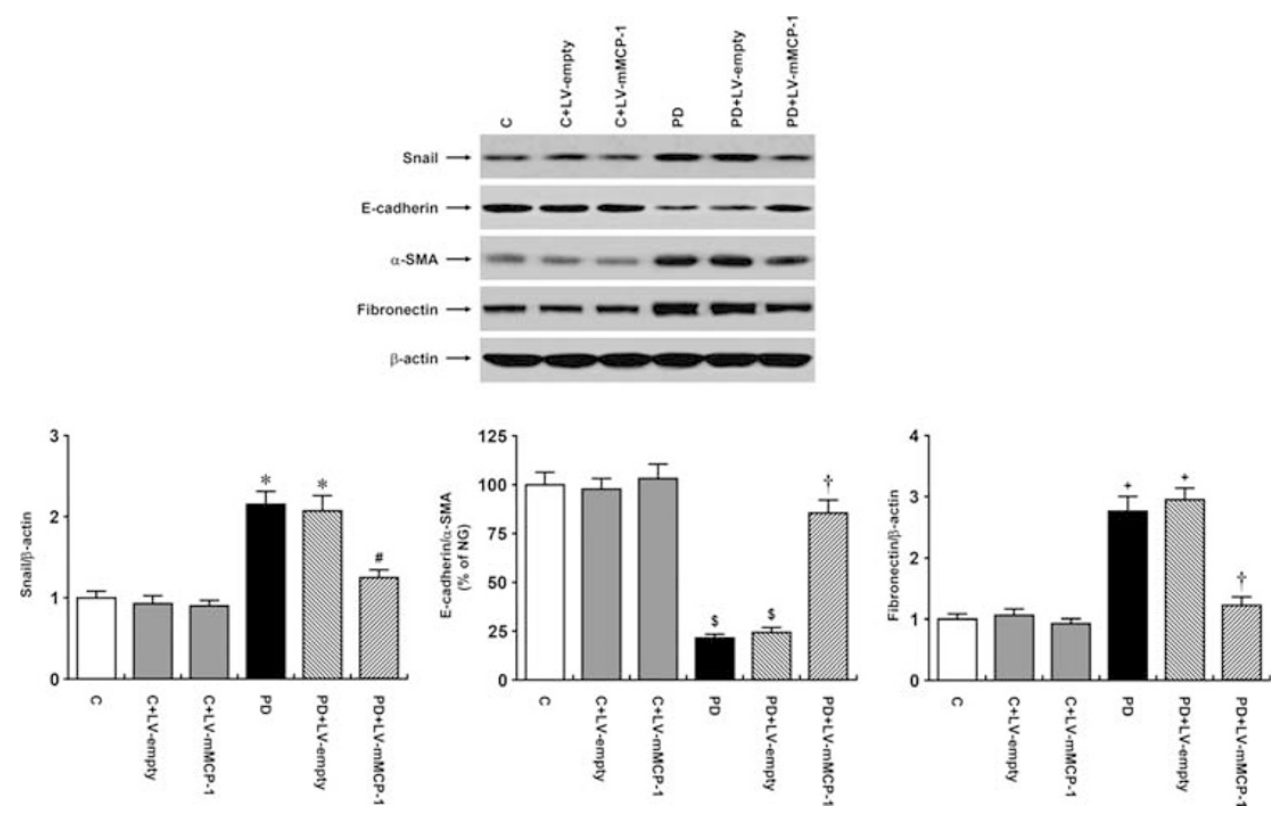

Figure 7 (a) Snail, E-cadherin, $\alpha$-SMA, and fibronectin protein expression in the peritoneum of control (C), C + empty lentivirus (C + LV-empty), $\mathrm{C}+$ lentivirus vector containing mMCP-1 (C + LV-mMCP-1), PD, PD + LV-empty, and PD + LV-mMCP-1 rats (a representative of five western blots). Snail, $\alpha$-SMA, and fibronectin protein expression was significantly increased, while E-cadherin protein expression was significantly decreased in rats treated with PD solution compared with $C$ rats, and these changes were significantly attenuated by LV-mMCP-1 but not by LV-empty. ${ }^{*} P<0.05$ vs $C,{ }^{\#} P<0.05$ vs $\mathrm{PD},{ }^{\$} P<0.005$ vs $C,{ }^{\dagger} P<0.01$ vs $\mathrm{PD},{ }^{+} P<0.01$ vs $C$. (b) Masson's trichrome staining and immunohistochemical staining for $\mathrm{E}-\mathrm{cadherin,} \alpha-\mathrm{SMA}$, and fibronectin in the peritoneum samples from control (C), C + empty lentivirus (C + LV-empty), C + lentivirus vector containing mMCP-1 (C + LV-mMCP-1), PD, PD + LV-empty, and PD + LV-mMCP-1 rats $(\times 200)$. Peritoneal fibrosis assessed by Masson's trichrome staining was significantly worse in PD rats than in $\mathrm{C}$ rats, and the extent of peritoneal fibrosis was significantly reduced by LV-mMCP-1 treatment. The intensity of E-cadherin staining was significantly lower, while $\alpha$-SMA and fibronectin staining intensities were significantly higher in PD compared with C rats, and LV-mMCP-1 treatment significantly abrogated these changes in PD rats. ${ }^{*} P<0.01$ vs $C,{ }^{\sharp} P<0.05$ vs $P D,{ }^{\$} P<0.001$ vs $C,{ }^{\dagger} P<0.01$ vs PD.

Figure 6 (a) MCP-1 and CCR2 protein expression in the peritoneum samples from control (C), C + empty lentivirus (C+ LV-empty), C + lentivirus vector containing mMCP-1 (C + LV-mMCP-1), PD, PD + LV-empty, and PD + LV-mMCP-1 rats (a representative of five western blots). Peritoneal MCP-1 expression was significantly increased in rats treated with PD solution compared with $\mathrm{C}$ rats, while there was no significant difference in CCR2 protein expression among the groups. (b) Immunohistochemical staining for MCP-1 in the peritoneum samples from control (C) and PD rats. In control rats, the staining for MCP-1 protein was weak and mainly localized in peritoneal mesothelial cells, while fibroblast-like cells and infiltrated inflammatory cells (arrow heads) were responsible for the increase in MCP-1 staining in PD rats $(\times 200$ and 400$)$. ${ }^{*} P<0.05$ vs $C$ rats. 
b
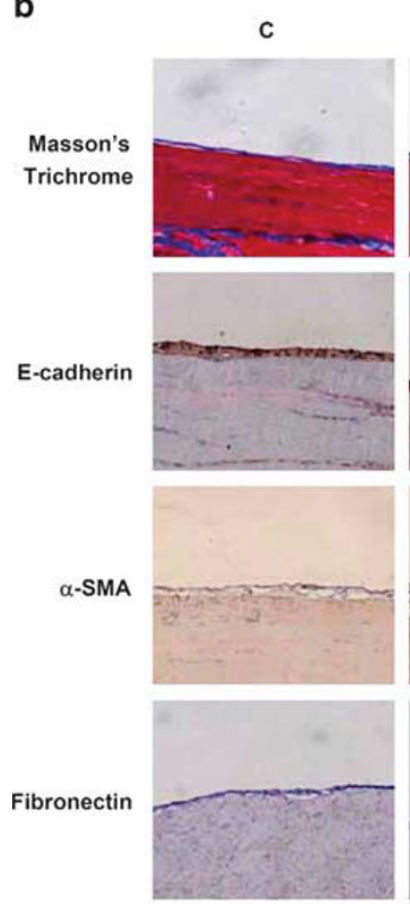

$\underset{\text { LV-empty }}{\text { C+ }}$
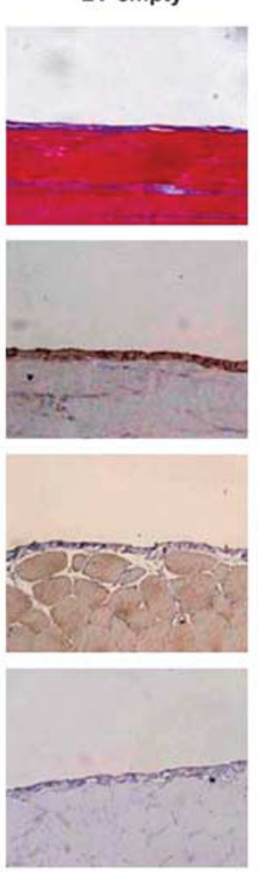

C+
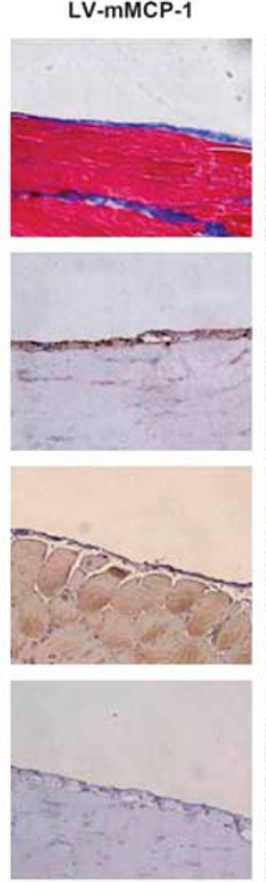

PD
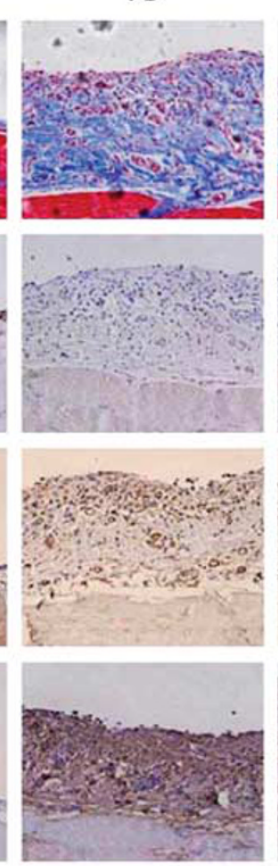

PD+
L-empty
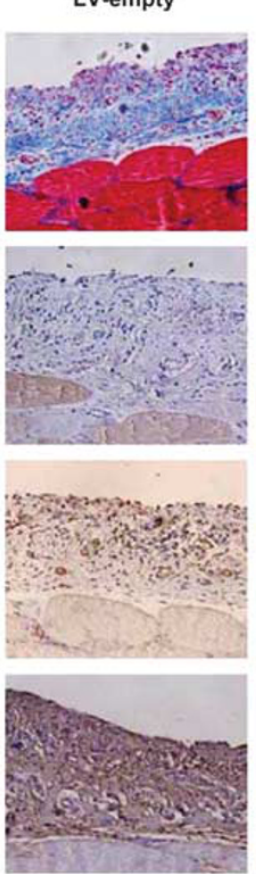

$\mathrm{PD}+$

LV-mMCP-1
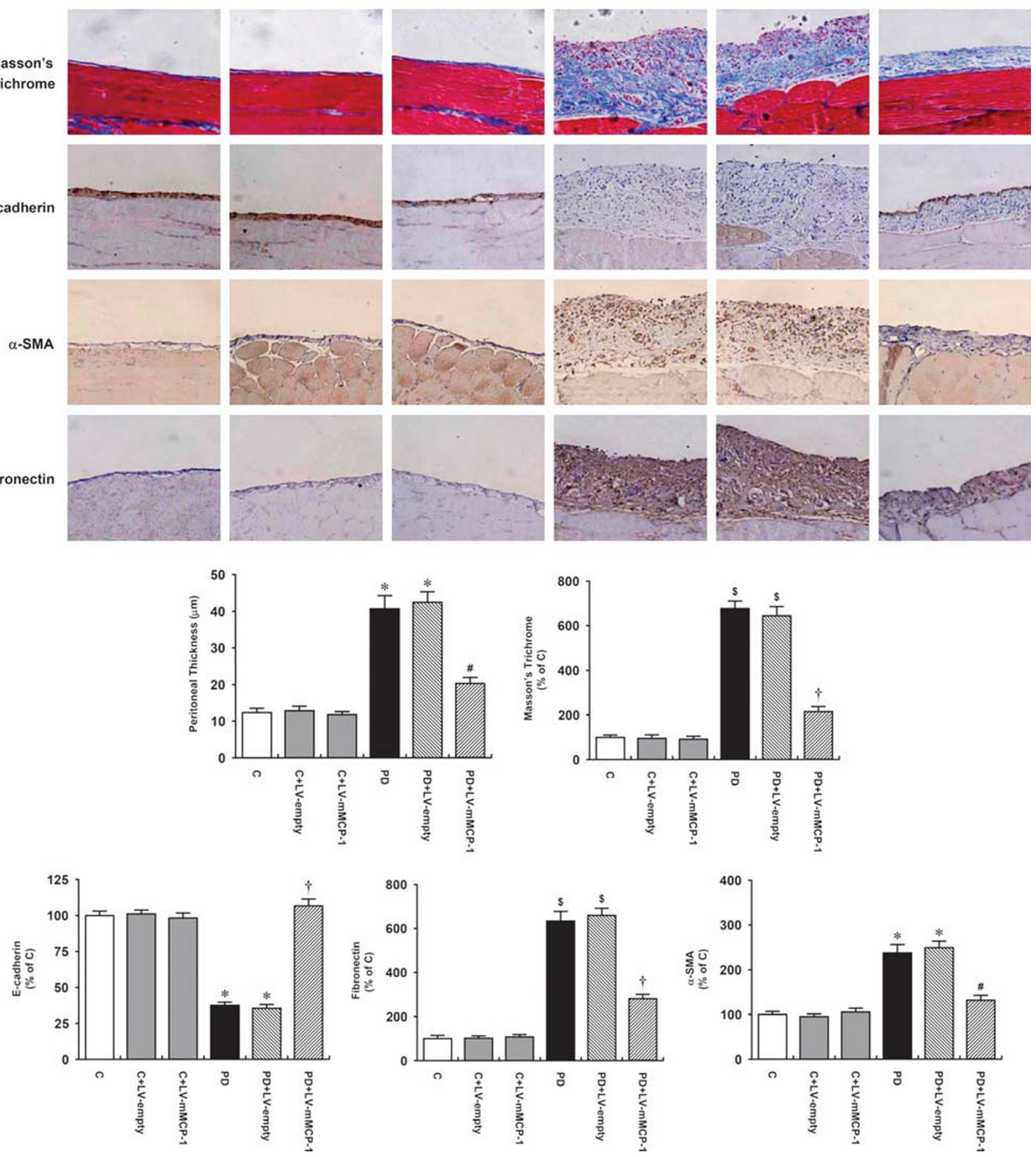

Figure 7 (Continued)

production. As aforementioned, since TGF- $\beta 1$ is the principal mediator of EMT, our results strongly suggest that the MCP-1/CCR2 system mediates PD-related EMT of PMCs and PF partly via the TGF- $\beta 1$ pathway (Figure 8 ).

To inhibit the MCP-1/CCR2 system, two different methods were used in the present study; mMCP-1, an N-terminal deletion mutant of the human MCP-1 gene (7ND), ${ }^{24}$ and RS102895, which is a specific chemical inhibitor of CCR2 and does not affect chemotaxis or postreceptor signaling of any kind, were used. However, RS102895 was used only in the in vitro but not in the in vivo experiments for several reasons. First, there was little information on the pharmacokinetics of 


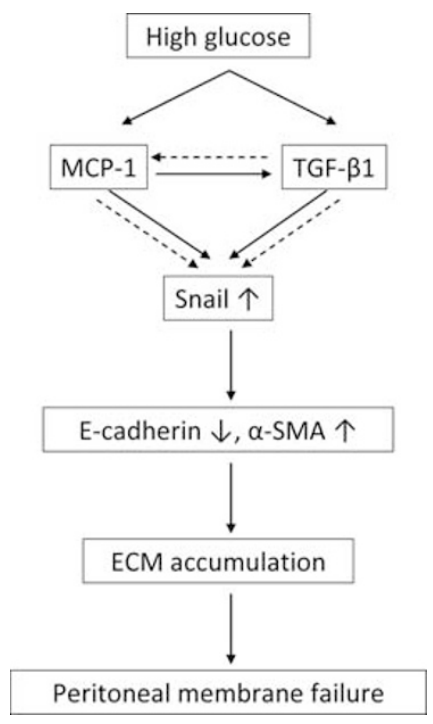

Figure 8 A schematic representation showing the outlined hypothesis.

intraperitoneally administered RS102895 and the recommended dose for intraperitoneal RS102895. Second, the expense of the total amount of RS102895 required for 4-week oral use in rats was not affordable. Third, intraperitoneal gene delivery with viral vector has been demonstrated to be efficient in previous studies. ${ }^{8,36}$ Fourth, we also have already set up the lentiviral delivery system successfully. ${ }^{37}$ In the current study, therefore, the in vivo experiments were performed using only mMCP-1 lentivirus.

In summary, MCP-1 directly decreased E-cadherin expression and increased $\alpha$-SMA and fibronectin expression via CCR2 in HPMCs, and the MCP-1/CCR2 system was involved in PD-related EMT and ECM accumulation via the TGF- $\beta 1$ pathway. These findings suggest that MCP-1 may act as a mediator of EMT as well as a profibrogenic cytokine in PMCs in addition to its role as an important mediator of monocyte recruitment.

\section{ACKNOWLEDGEMENTS}

This study was supported by Basic Science Research Program through the National Research Foundation of Korea (NRF) funded by the Ministry of Education, Science, and Technology (2011-0027214), the BK21 (Brain Korea 21) Project for Medical Sciences, Yonsei University, and the National Research Foundation of Korea (NRF) grant funded by the Korea government (MEST) (No. 2011-0030711).

\section{DISCLOSURE/CONFLICT OF INTEREST}

The authors declare no conflict of interest.

1. Nesrallah G, Mendelssohn DC. Modality options for renal replacement therapy: the integrated care concept revisited. Hemodial Int 2006;10:143-151.

2. Krediet RT, Lindholm B, Rippe B. Pathophysiology of peritoneal membrane failure. Perit Dial Int 2000;20(Suppl 4):S22-S42.

3. Dobbie JW. Pathogenesis of peritoneal fibrosing syndromes (sclerosing peritonitis) in peritoneal dialysis. Perit Dial Int 1992;12: $14-27$.
4. Yanez-Mo M, Lara-Pezzi E, Selgas $\mathrm{R}$, et al. Peritoneal dialysis and epithelial-to-mesenchymal transition of mesothelial cells. N Engl J Med 2003:348:403-413.

5. Kalluri R, Weinberg RA. The basics of epithelial-mesenchymal transition. J Clin Invest 2009;119:1420-1428.

6. Aroeira LS, Aguilera A, Sanchez-Tomero JA, et al. Epithelial to mesenchymal transition and peritoneal membrane failure in peritoneal dialysis patients: pathologic significance and potential therapeutic interventions. J Am Soc Nephrol 2007;18: 2004-2013.

7. Yang $\mathrm{AH}, \mathrm{Chen} \mathrm{JY}$, Lin JK. Myofibroblastic conversion of mesothelial cells. Kidney Int 2003;63:1530-1539.

8. Margetts PJ, Bonniaud P, Liu L, et al. Transient overexpression of TGF-\{beta\}1 induces epithelial mesenchymal transition in the rodent peritoneum. J Am Soc Nephrol 2005;16:425-436.

9. Segerer S, Nelson PJ, Schlondorff D. Chemokines, chemokine receptors, and renal disease: from basic science to pathophysiologic and therapeutic studies. J Am Soc Nephrol 2000;11:152-176.

10. Chow FY, Nikolic-Paterson DJ, Ozols E, et al. Monocyte chemoattractant protein-1 promotes the development of diabetic renal injury in streptozotocin-treated mice. Kidney Int 2006;69:73-80.

11. Wada T, Furuichi K, Sakai N, et al. Gene therapy via blockade of monocyte chemoattractant protein-1 for renal fibrosis. J Am Soc Nephrol 2004;15:940-948.

12. $\mathrm{Xu}$ J, Lin SC, Chen J, et al. CCR2 mediates the uptake of bone marrowderived fibroblast precursors in angiotensin II-induced cardiac fibrosis. Am J Physiol Heart Circ Physiol 2011;301:H538-H547.

13. Topley N, Liberek T, Davenport A, et al. Activation of inflammation and leukocyte recruitment into the peritoneal cavity. Kidney Int Suppl 1996;56:S17-S21.

14. Giunti S, Pinach S, Arnaldi L, et al. The MCP-1/CCR2 system has direct proinflammatory effects in human mesangial cells. Kidney Int 2006;69:856-863.

15. Urushihara M, Ohashi N, Miyata K, et al. Addition of angiotensin Il type 1 receptor blocker to CCR2 antagonist markedly attenuates crescentic glomerulonephritis. Hypertension 2011;57:586-593.

16. Kang $\mathrm{YS}$, Lee $\mathrm{MH}$, Song $\mathrm{HK}$, et al. CCR2 antagonism improves insulin resistance, lipid metabolism, and diabetic nephropathy in type 2 diabetic mice. Kidney Int 2010;78:883-894.

17. Park J, Ryu DR, Li JJ, et al. MCP-1/CCR2 system is involved in high glucose-induced fibronectin and type IV collagen expression in cultured mesangial cells. Am J Physiol Renal Physiol 2008;295: F749-F757.

18. Nam BY, Paeng J, Kim SH, et al. The MCP-1/CCR2 axis in podocytes is involved in apoptosis induced by diabetic conditions. Apoptosis 2012;17:1-13.

19. Giunti S, Tesch GH, Pinach S, et al. Monocyte chemoattractant protein-1 has prosclerotic effects both in a mouse model of experimental diabetes and in vitro in human mesangial cells. Diabetologia 2008;51:198-207.

20. Schneider A, Panzer U, Zahner G, et al. Monocyte chemoattractant protein-1 mediates collagen deposition in experimental glomerulonephritis by transforming growth factor-beta. Kidney Int 1999;56: 135-144.

21. Wolf G, Jocks $T$, Zahner $G$, et al. Existence of a regulatory loop between MCP-1 and TGF-beta in glomerular immune injury. Am J Physiol Renal Physiol 2002;283:F1075-F1084.

22. Gruden G, Setti G, Hayward A, et al. Mechanical stretch induces monocyte chemoattractant activity via an NF-kappaB-dependent monocyte chemoattractant protein-1-mediated pathway in human mesangial cells: inhibition by rosiglitazone. J Am Soc Nephrol 2005; 16:688-696.

23. Stylianou $E_{\text {, Jenner } L A}$, Davies $M$, et al. Isolation, culture and characterization of human peritoneal mesothelial cells. Kidney Int 1990:37:1563-1570.

24. Zhang YJ, Rutledge BJ, Rollins BJ. Structure/activity analysis of human monocyte chemoattractant protein-1 (MCP-1) by mutagenesis. Identification of a mutated protein that inhibits MCP-1-mediated monocyte chemotaxis. J Biol Chem 1994;269: $15918-15924$.

25. Musi B, Braide M, Carlsson O, et al. Biocompatibility of peritoneal dialysis fluids: long-term exposure of nonuremic rats. Perit Dial Int 2004;24:37-47. 
26. Loureiro J, Schilte M, Aguilera A, et al. BMP-7 blocks mesenchymal conversion of mesothelial cells and prevents peritoneal damage induced by dialysis fluid exposure. Nephrol Dial Transplant 2010;25: 1098-1108.

27. Lv ZD, $\mathrm{Na} \mathrm{D}, \mathrm{Ma} X \mathrm{XY}$, et al. Human peritoneal mesothelial cell transformation into myofibroblasts in response to TGF-ss1 in vitro. Int J Mol Med 2011;27:187-193.

28. Shook D, Keller R. Mechanisms, mechanics and function of epithelialmesenchymal transitions in early development. Mech Dev 2003;120: 1351-1383.

29. Xue C, Plieth D, Venkov C, et al. The gatekeeper effect of epithelialmesenchymal transition regulates the frequency of breast cancer metastasis. Cancer Res 2003;63:3386-3394.

30. Zeisberg $M$, Kalluri R. The role of epithelial-to-mesenchymal transition in renal fibrosis. J Mol Med (Berl) 2004;82:175-181.

31. Wynn TA. Cellular and molecular mechanisms of fibrosis. J Patho 2008;214:199-210.

32. Hung KY, Huang JW, Chiang CK, et al. Preservation of peritoneal morphology and function by pentoxifylline in a rat model of peritoneal dialysis: molecular studies. Nephrol Dial Transplant 2008;23: 3831-3840.

33. Zareie $\mathrm{M}$, De Vriese AS, Hekking $\mathrm{LH}$, et al. Immunopathological changes in a uraemic rat model for peritoneal dialysis. Nephrol Dial Transplant 2005;20:1350-1361.

34. Fabbrini $P$, Schilte $M N$, Zareie $M$, et al. Celecoxib treatment reduces peritoneal fibrosis and angiogenesis and prevents ultrafiltration failure in experimental peritoneal dialysis. Nephrol Dial Transplant 2009;24: 3669-3676.

35. Soria G, Ofri-Shahak M, Haas I, et al. Inflammatory mediators in breast cancer: coordinated expression of TNFalpha \& IL-1beta with CCL2 \& CCL5 and effects on epithelial-to-mesenchymal transition. BMC Cancer 2011;11:130

36. Yu MA, Shin KS, Kim JH, et al. HGF and BMP-7 ameliorate high glucoseinduced epithelial-to-mesenchymal transition of peritoneal mesothelium. J Am Soc Nephrol 2009;20:567-581.

37. Kim DK, Nam BY, Li JJ, et al. Translationally controlled tumour protein is associated with podocyte hypertrophy in a mouse model of type 1 diabetes. Diabetologia 2012;55:1205-1217. 